DESY 98-166

UCRHEP-T242

January 1999

\title{
Neutrino Masses in Supersymmetry: R-Parity and Leptogenesis
}

\author{
Ernest $\mathrm{Ma}^{1}$, Martti Raidal ${ }^{2}$, and Utpal Sarkar ${ }^{3}$ \\ ${ }^{1}$ Department of Physics, University of California \\ Riverside, California 92521, USA \\ 2 Theory Group, DESY, D-22603 Hamburg, Germany \\ 3 Physical Research Laboratory, Ahmedabad 380 009, India
}

In the supersymmetric standard model of particle interactions, R-parity nonconservation is often invoked to obtain nonzero neutrino masses. We point out here that such interactions of the supersymmetric particles would erase any pre-existing lepton or baryon asymmetry of the universe before the electroweak phase transition through the $B+L$ violating sphaleron processes. We then show how neutrino masses may be obtained in supersymmetry (assuming R-parity conservation) together with successful leptogenesis and predict the possible existence of new observable particles. 
Two issues in particle physics are critically important today. One is the possible existence of neutrino masses, as evidenced by the ongoing excitement generated by the recent report of atmospheric neutrino oscillations [1], as well as previous other indications of solar [2] and accelerator [3] neutrino oscillations. The other is the possible existence of supersymmetry, as evidenced by the enormous, continuing efforts of both experimentalists and theorists in devising ways of searching for the predicted new particles in existing and future highenergy colliders [4. In the minimal standard model (SM) of quarks and leptons without supersymmetry, neutrinos are massless. To make them massive, new physics have to be assumed [5]. In the minimal supersymmetric standard model (MSSM) which assumes Rparity conservation, neutrinos are also massless. To make them massive, many previous discussions have been based on R-parity nonconservation [6]. We point out here one very important consequence of this hypothesis, namely that there are now unavoidable leptonnumber violating interactions at the supersymmetry breaking scale. Combining these with the $B+L$ violating sphaleron processes [7], any pre-existing $B$ or $L$ or $B-L$ asymmetry of the universe would be erased [8,9]. This is so unless $B-3 L_{i}$ is conserved [10, [1] even after the electroweak phase transition, which is of course not the case here. A more desirable mechanism for neutrino masses in supersymmetry should be such that leptogenesis [12] would be also possible in the same context.

There are two appealing mechanisms for neutrino masses which are intimately related to successful leptogenesis. One is the canonical seesaw mechanism [13], in which heavy Majorana singlet neutrinos may decay to generate a lepton asymmetry [14], which gets converted into the present observed baryon asymmetry through the electroweak phase transition. The other is to have neutrino masses as well as leptogenesis through heavy Higgs triplets [15]. Both are conceived originally as simple extensions of the SM, but they are also applicable as simple extensions of the MSSM. The key in both cases is that lepton-number violation occurs 
at a mass scale many orders of magnitude greater than the electroweak breaking scale of $10^{2}$ $\mathrm{GeV}$. In models of R-parity violation, the participating particles are those of the MSSM, hence lepton-number violation is a fast process at the supersymmetry breaking scale of $10^{3}$ $\mathrm{GeV}$. We show in the following that for realistic neutrino masses, such models do not allow leptogenesis. Included in this class of models are those which obtain neutrino masses from a radiative mechanism with suppressed Yukawa couplings, such as the Zee model 16 18]. We then propose a specific supersymmetric extension of the Zee model with unsuppressed Yukawa couplings which has new particles at a much higher mass scale. We demonstrate the possibility of obtaining realistic radiative neutrino masses as well as successful leptogenesis in this model. It also contains other new particles that should be light enough for discovery at future accelerators such as the planned Large Hadron Collider (LHC) at CERN.

In the MSSM, R-parity of a particle is defined as

$$
R \equiv(-1)^{3 B+L+2 J},
$$

where $B$ is its baryon number, $L$ its lepton number, and $J$ its spin angular momentum. Hence the SM particles have $R=+1$ and their supersymmetric partners have $R=-1$. Using the common notation where all chiral superfields are considered left-handed, the three families of leptons and quarks are given by

$$
\begin{gathered}
L_{i}=\left(\nu_{i}, e_{i}\right) \sim(1,2,-1 / 2), \quad e_{i}^{c} \sim(1,1,1), \\
Q_{i}=\left(u_{i}, d_{i}\right) \sim(3,2,1 / 6), \quad u_{i}^{c} \sim\left(3^{*}, 1,-2 / 3\right), \quad d_{i}^{c} \sim\left(3^{*}, 1,1 / 3\right),
\end{gathered}
$$

where $i$ is the family index, and the two Higgs doublets are given by

$$
H_{1}=\left(h_{1}^{0}, h_{1}^{-}\right) \sim(1,2,-1 / 2), \quad H_{2}=\left(h_{2}^{+}, h_{2}^{0}\right) \sim(1,2,1 / 2),
$$

where the $S U(3)_{C} \times S U(2)_{L} \times U(1)_{Y}$ content of each superfield is also indicated. If R-parity is conserved, the superpotential is restricted to have only the terms 


$$
W=\mu H_{1} H_{2}+f_{i j}^{e} H_{1} L_{i} e_{j}^{c}+f_{i j}^{d} H_{1} Q_{i} d_{j}^{c}+f_{i j}^{u} H_{2} Q_{i} u_{j}^{c} .
$$

If R-parity is violated but not baryon number, then the superpotential contains the additional terms

$$
W^{\prime}=\epsilon_{i} L_{i} H_{2}+\lambda_{i j k} L_{i} L_{j} e_{k}^{c}+\lambda_{i j k}^{\prime} L_{i} Q_{j} d_{k}^{c},
$$

resulting in nonzero neutrino masses either from mixing with the neutralino mass matrix [6] or in one-loop order 19.

If lepton-number violating interactions such as

$$
L_{i}+Q_{j} \rightarrow\left(\tilde{d}_{k}^{c}\right)^{*} \rightarrow H_{1}+Q_{l}
$$

are in equilibrium in the early universe, any pre-existing lepton asymmetry would be erased. To make sure that this does not happen, the following condition has to be satisfied:

$$
\frac{\lambda^{\prime 2} T}{8 \pi} \lesssim 1.7 \sqrt{g_{*}} \frac{T^{2}}{M_{P}} \quad \text { at } T=M_{S U S Y}
$$

where $g_{*} \sim 10^{2}$ is the effective number of interacting relativistic degrees of freedom and $M_{P} \sim 10^{19} \mathrm{GeV}$ is the Planck mass. Assuming that the supersymmetry breaking scale $M_{S U S Y}$ is $10^{3} \mathrm{GeV}$, we find

$$
\lambda^{\prime} \lesssim 2 \times 10^{-7},
$$

which is very much below the typical minimum value of $10^{-4}$ needed for radiative neutrino masses [20]. A similar bound was presented from dimensional arguments [8]. Larger values of $\lambda^{\prime}$ are allowed if there is a conserved $\left(B-3 L_{i}\right)$ symmetry [10]. However, there would be other severe phenomenological restrictions in that case [21]. The bound of Eq. (9) cannot be evaded even if one uses the bilinear term in Eq. (6) for neutrino masses instead, because the 
induced mixing would change Eq. (5) and introduce trilinear couplings which violate lepton number and an effective $\lambda^{\prime}$ is unavoidable.

Because the $B+L$ violating sphaleron processes are effective at temperatures from $10^{2}$ to $10^{12} \mathrm{GeV}$, the presence of the above $L$ violating interactions would also erase any preexisting $B$ or $B-L$ asymmetry of the universe before the electroweak phase transition. To have the successful conversion of a pre-existing $B$ or $L$ or $B-L$ asymmetry into the present observed baryon asymmetry of the universe, it is necessary that the lepton-number violating interactions of Eq. (6) be suppressed. This means that although R-parity violation may exist, it will mostly be negligible phenomenologically. In particular, it will not contribute significantly to neutrino masses.

It is clear from the above discussion that we need to increase the mass scale of any appreciable lepton-number violating interaction for it to be consistent with leptogenesis. Of course we would also like it to generate appropriate neutrino masses. As remarked earlier, such models [14, 15] are already well-known. Whether heavy Majorana singlet neutrinos or heavy Higgs triplets are used, the scale of lepton-number violation is determined by their masses, which may be greater than $10^{7} \mathrm{GeV}$ or $10^{13} \mathrm{GeV}$ in the case of the former or the latter respectively. In both cases, there are no new observable particles or interactions below that scale. Extending these models to include supersymmetry [22] does not change the above conclusion, other than the obvious fact that the SM now becomes the MSSM. Indeed it should be noted that R-parity is conserved in both such extensions, because lepton number is violated only by two units. On the other hand, models of radiative neutrino masses often include new particles which are amenable to discovery at planned future accelerators. They may also offer the possibility of naturally large mixing angles which are required for atmospheric neutrino oscillations and for vacuum solar neutrino oscillations.

In models of radiative neutrino masses [5,23], in addition to the suppression due to the 
$1 / 16 \pi^{2}$ factor of each loop, there is often another source of suppression due to the Yukawa couplings involved. In the supersymmetric case with R-parity violation [19], the suppression is proportional to a quadratic combination of charged-lepton or down-quark masses [20]. That is the reason why $\lambda$ and $\lambda^{\prime}$ of Eq. (6) cannot be too small. In the original Zee model [16], the SM is extended to include a charged scalar $\chi^{+}$and a second Higgs doublet. The radiative mechanism for generating neutrino masses is exactly the same [5] as given by Eq. (6) with $e^{c}$ replaced by $\chi^{+}$. Although the mass of $\chi^{+}$is not constrained by $M_{S U S Y}$, the previously mentioned Yukawa suppression factor remains, hence it cannot be too large or else neutrino masses would be too small, as shown below.

The relevant terms of the interaction Lagrangian are given by

$$
\mathcal{L}=\sum_{i<j} f_{i j}\left(\nu_{i} e_{j}-e_{i} \nu_{j}\right) \chi^{+}+\mu\left(\phi_{1}^{+} \phi_{2}^{0}-\phi_{1}^{0} \phi_{2}^{+}\right) \chi^{-}+H . c .,
$$

where two Higgs doublets are needed or else there would be no $\phi \phi \chi$ coupling. Lepton number is violated in the above by two units, hence we expect the realization of an effective dimension-five operator $\Lambda^{-1} \phi^{0} \phi^{0} \nu_{i} \nu_{j}$ for naturally small Majorana neutrino masses [5]. This occurs here in one loop and the elements of the $3 \times 3$ neutrino mass matrix are given by

$$
\left(m_{\nu}\right)_{i j}=f_{i j}\left(m_{i}^{2}-m_{j}^{2}\right)\left(\frac{\mu v_{2}}{v_{1}}\right) F\left(m_{\chi}^{2}, m_{\phi_{1}}^{2}\right),
$$

where $v_{1,2} \equiv\left\langle\phi_{1,2}^{0}\right\rangle$ and $m_{i}$ are the charged-lepton masses which come from $\phi_{1}$ but not $\phi_{2}$. The function $F$ is given by

$$
F\left(m_{1}^{2}, m_{2}^{2}\right)=\frac{1}{16 \pi^{2}} \frac{1}{m_{1}^{2}-m_{2}^{2}} \ln \frac{m_{1}^{2}}{m_{2}^{2}} .
$$

Since the $m_{\tau}^{2}$ terms in Eq. (11) are likely to be dominant, this model has two nearly massdegenerate neutrinos which mix maximally [17,24]. This is very suitable for explaining the atmospheric neutrino data 回, but only in conjunction with the LSND data [3]. Let $m_{\chi}=1$ $\mathrm{TeV}, m_{\phi_{1}}=100 \mathrm{GeV}, \mu=100 \mathrm{GeV}, v_{2} / v_{1}=1$, and $f_{\mu \tau}=f_{e \tau}=10^{-7}$ to satisfy Eq. (9), then 
the $m_{\tau}^{2}$ terms generate a neutrino mass of $0.0013 \mathrm{eV}$, which is very much below the necessary $1 \mathrm{eV}$ or so indicated by the LSND data. We note that Eq. (8) constrains the combination $f^{2} / m_{\chi}$, whereas $m_{\nu}$ goes like $f / m_{\chi}^{2}$. Hence neutrino masses would only decrease if we increase $m_{\chi}$. As long as Eq. (11) gets a suppression from $m_{\tau}^{2}$ (which comes of course from the Yukawa coupling $\left.m_{\tau} / v_{1}\right)$, the conflict with leptogenesis is a real problem.

We now propose a new supersymmetric variation of the Zee model which has a fourth family of leptons with unsuppressed Yukawa couplings for generating neutrino masses (the fourth quark family should be also added to cancel anomalies but we do not consider their phenomenology here). This model preserves R-parity and the scale of lepton-number violation by two units is of order $10^{13} \mathrm{GeV}$, which is suitable for leptogenesis. We add to the MSSM the new superfields shown in Table I. The discrete $Z_{2}$ symmetry is just the usual one for defining R-parity; i.e. the quark and lepton superfields are odd and the Higgs superfields are even. The discrete $Z_{2}^{\prime}$ symmetry is new and it distinguishes the new particles of Table I from those of the MSSM which are assumed to be even.

The relevant terms in the R-parity preserving superpotential of this model are given by

$$
\begin{aligned}
W= & \mu_{12}\left(h_{1}^{0} h_{2}^{0}-h_{1}^{-} h_{2}^{+}\right)+\mu_{34}\left(h_{3}^{0} h_{4}^{0}-h_{3}^{-} h_{4}^{+}\right)+m_{\chi} \chi_{1}^{+} \chi_{2}^{-}+\left(m_{E} / v_{1}\right)\left(h_{1}^{0} E^{-}-h_{1}^{-} N_{1}^{0}\right) E^{+} \\
& +f_{i}\left(\nu_{i} h_{3}^{-}-e_{i}^{-} h_{3}^{0}\right) E^{+}+f_{j}^{\prime}\left(\nu_{j} E^{-}-e_{j}^{-} N_{1}^{0}\right) \chi_{1}^{+}+f_{24}\left(h_{2}^{+} h_{4}^{0}-h_{2}^{0} h_{4}^{+}\right) \chi_{2}^{-},
\end{aligned}
$$

and $v_{1,2}$ are the vacuum expectation values of $h_{1,2}^{0}$. The unsuppressed one-loop diagram generating neutrino masses is shown in Fig. 1. We note that the effective dimension-five operator $L_{i} L_{j} H_{2} H_{2}$ is indeed realized. Assuming that the masses of the scalar leptons of the fourth family to be equal to $M_{S U S Y}$, we find

$$
\left(m_{\nu}\right)_{i j}=\frac{\left(f_{i} f_{j}^{\prime}+f_{i}^{\prime} f_{j}\right) f_{24} v_{2}^{2} m_{E} \mu_{12} \mu_{34}}{16 \pi^{2} v_{1} M_{S U S Y}^{2} m_{\chi}} \ln \frac{m_{\chi}^{2}}{M_{S U S Y}^{2}} .
$$

To get an estimate of the above expression, let $f_{i}=f_{j}^{\prime}=f_{24}=1, m_{E}=v_{1}, \mu_{12}=\mu_{34}=$ $M_{S U S Y}$, then 


$$
m_{\nu}=\frac{1}{8 \pi^{2}} \frac{v_{2}^{2}}{m_{\chi}} \ln \frac{m_{\chi}^{2}}{M_{S U S Y}^{2}}
$$

Assuming $v_{2} \sim 10^{2} \mathrm{GeV}, m_{\chi} \sim 10^{13} \mathrm{GeV}$, and $M_{S U S Y} \sim 10^{3} \mathrm{GeV}$, we get $m_{\nu} \sim 0.6 \mathrm{eV}$. This is just one order of magnitude greater than the square root of the $\Delta m^{2} \sim 5 \times 10^{-3} \mathrm{eV}^{2}$ needed for atmospheric neutrino oscillations [1]. Reducing slightly the above dimensionless couplings from unity would fit the data quite well. Since $m_{\chi} \sim 10^{13} \mathrm{GeV}$ is now allowed, leptogenesis should be possible as demonstrated in [9]. It was argued [25] that due to the gravitino production constraints on the reheating temperature after the inflation such a high leptogenesis scale is allowed only in models with small gravitino masses, e.g., models with gauge mediated SUSY breaking. However, new efficient reheating mechanisms [26] allow production of particles with such masses, and consequently leptogenesis, without exponential suppression.

It has recently been shown [27] that the structure of Eq. (14) for the $\mu-\tau$ sector is naturally suited for the large mixing solution of atmospheric neutrino oscillations. To be more specific, the $2 \times 2$ submatrix of Eq. (14) for the $\mu-\tau$ sector can be written as

$$
\mathcal{M}=m_{0}\left[\begin{array}{cc}
2 \sin \alpha \sin \alpha^{\prime} & \sin \left(\alpha+\alpha^{\prime}\right) \\
\sin \left(\alpha+\alpha^{\prime}\right) & 2 \cos \alpha \cos \alpha^{\prime}
\end{array}\right],
$$

where $\tan \alpha=f_{\mu} / f_{\tau}$ and $\tan \alpha^{\prime}=f_{\mu}^{\prime} / f_{\tau}^{\prime}$. The eigenvalues of $\mathcal{M}$ are then given by $m_{0}\left(c_{1} \pm 1\right)$, where $c_{1}=\cos \left(\alpha-\alpha^{\prime}\right)$, and the effective $\sin ^{2} 2 \theta$ for $\nu_{\mu}-\nu_{\tau}$ oscillations is $\left(1-c_{2}\right) /\left(1+c_{2}\right)$, where $c_{2}=\cos \left(\alpha+\alpha^{\prime}\right)$. If we choose $\tan \alpha \sim \tan \alpha^{\prime} \sim 1$, then $c_{1} \sim 1$ and $c_{2} \sim 0$. In that case, maximal mixing between a heavy $\left(2 m_{0}\right)$ and a light $\left(s_{1}^{2} m_{0} / 2\right)$ neutrino occurs as an explanation of the atmospheric data. If we assume further that $f_{e}<<f_{\mu, \tau}$ and $f_{e}^{\prime}<<f_{\mu, \tau}^{\prime}$, then the small-angle matter-enhanced solution of solar neutrino oscillations may be obtained as well.

Our proposed model has the twin virtues of an acceptable neutrino mass matrix given by Eq. (14) and the possibility of generating a lepton asymmetry of the universe through the 
decays of $\chi_{1,2}^{ \pm}$. It is also phenomenologically safe because all the additions to the SM do not alter its known successes. Neither the fourth family of leptons $E^{ \pm}, N_{1,2}^{0}$ nor the two extra Higgs doublets $H_{3,4}$ mix with their SM analogs because they are odd under the new discrete $Z_{2}^{\prime}$ symmetry. In particular, $H_{3}$ and $H_{4}$ do not couple to the known quarks and leptons, hence flavor-changing neutral currents are suppressed here as in the SM. The lepton-number violation of this model is associated with $m_{\chi}$ which is of order $10^{13} \mathrm{GeV}$. However, the fourth family of leptons should have masses of order $10^{2} \mathrm{GeV}$ and be observable at planned future colliders. The two extra Higgs doublets should also be observable with an energy scale of order $M_{S U S Y}$. The soft supersymmetry-breaking terms of this model are assumed to break $Z_{2}^{\prime}$ without breaking $Z_{2}$. Hence there will still be a stable LSP (lightest supersymmetric particle) and a fourth-family lepton will still decay into ordinary leptons. For example, because $\tilde{h}_{3}^{0}$ mixes with $\tilde{h}_{1}^{0}$, the decay

$$
E^{-} \rightarrow \mu^{-} \tilde{h}_{3}^{0}\left(\tilde{h}_{1}^{0}\right) \rightarrow \mu^{-} \tau^{+} \tau^{-}
$$

is possible and would make a spectacular signature.

In conclusion, we have shown in this paper that R-parity violation in supersymmetry is generically inconsistent with leptogenesis because the lepton-number violating interactions would act in conjunction with the $B+L$ violating sphaleron processes and erase any preexisting $B$ or $L$ or $B-L$ asymmetry of the universe. This constraint means that any Rparity violation must be very small, so that it is of negligible phenomenological interest and cannot contribute significantly to neutrino masses. This conclusion also applies to models of radiative neutrino masses with suppressed Yukawa couplings, such as the Zee model. However, we have also shown that realistic radiative neutrino masses in supersymmetry are possible beyond the MSSM with R-parity conservation where the lepton-number violation is by two units and occurs at the mass scale of $10^{13} \mathrm{GeV}$. Our specific model (which is an extension of the Zee model with unsuppressed Yukawa couplings) also predicts new particles 
which should be observable in the future at the LHC.

\section{ACKNOWLEDGMENTS}

We thank W. Buchmüller for useful comments on the manuscript. The work of EM was supported in part by the U. S. Department of Energy under Grant No. DE-FG03-94ER40837, and MR acknowledges financial support from the Alexander von Humboldt Foundation.

[1] Y. Fukuda et al., Phys. Lett. B433, 9 (1998); B436, 33 (1998); Phys. Rev. Lett. 81, 1562 (1998).

[2] R. Davis, Prog. Part. Nucl. Phys. 32, 13 (1994); Y. Fukuda etal., Phys. Rev. Lett. 77, 1683 (1996); 81, 1158 (1998); P. Anselmann etal., Phys. Lett. B357, 237 (1995); B361, 235 (1996); J. N. Abdurashitov et al., Phys. Lett. B328, 234 (1994).

[3] C. Athanassopoulos et al., Phys. Rev. Lett. 75, 2650 (1995); 77, 3082 (1996); 81, 1774 (1998).

[4] For a recent review and a list of references, see for example V. Barger, hep-ph/9801440.

[5] For a recent overview, see E. Ma, Phys. Rev. Lett. 81, 1171 (1998).

[6] For a recent review, see for example J. W. F. Valle, hep-ph/9808292.

[7] V. A. Kuzmin, V. A. Rubakov, and M. E. Shaposhnikov, Phys. Lett. 155B, 36 (1985).

[8] B. A. Campbell, S. Davidson, J. E. Ellis and K. Olive, Phys. Lett. B256, 457 (1991).

[9] E. Ma, M. Raidal, and U. Sarkar, hep-ph/9811240.

[10] H. Dreiner and G. G. Ross, Nucl. Phys. B410, 188 (1993); A. Ilakovac and A. Pilaftsis, Nucl. Phys. B437, 491 (1995).

[11] E. Ma and U. Sarkar, Phys. Lett. B439, 95 (1998).

[12] For recent reviews, see for example, U. Sarkar, hep-ph/9809209; W. Buchmüller, hepph/9812447, and references therein. 
[13] M. Gell-Mann, P. Ramond, and R. Slansky, in Supergravity, edited by P. van Nieuwenhuizen and D. Z. Freedman (North-Holland, Amsterdam, 1979), p. 315; T. Yanagida, in Proceedings of the Workshop on the Unified Theory and the Baryon Number in the Universe, edited by O. Sawada and A. Sugamoto (KEK Report No. 79-18, Tsukuba, Japan, 1979), p. 95; R. N. Mohapatra and G. Senjanovic, Phys. Rev. Lett. 44, 1316 (1980).

[14] M. Fukugita and T. Yanagida, Phys. Lett. B174, 45 (1986).

[15] E. Ma and U. Sarkar, Phys. Rev. Lett. 80, 5716 (1998).

[16] A. Zee, Phys. Lett. 93B, 389 (1980).

[17] A. Yu. Smirnov and M. Tanimoto, Phys. Rev. D55, 1665 (1997).

[18] C. Jarlskog, M. Matsuda, S. Skadhauge, and M. Tanimoto, hep-ph/9812282.

[19] L. Hall and M. Suzuki, Nucl. Phys. B231, 419 (1984).

[20] For a recent discussion, see for example M. Drees, S. Pakvasa, X. Tata, and T. ter Veldhuis, Phys. Rev. D57, R5340 (1998).

[21] E. Ma, Phys. Lett. B433, 74 (1998); E. Ma and D. P. Roy, Phys. Rev. D58, 095005 (1998)..

[22] See for example, M. Plümacher, Nucl. Phys. B530, 207 (1998); G. Lazarides and Q. Shafi, Phys. Rev. D58, 071702 (1998); Q. Shafi and Z. Tavartkiladze, hep-ph/9811463.

[23] K. S. Babu and E. Ma, Mod. Phys. Lett. A4, 1975 (1989).

[24] N. Gaur, A. Ghosal, E. Ma, and P. Roy, Phys. Rev. D58, 071301 (1998).

[25] D. Delepine and U. Sarkar, hep-ph/9811479.

[26] G. Felder, L. Kofman, and A. Linde, hep-ph/9812289.

[27] E. Ma, hep-ph/9807386 (Phys. Lett. B, in press). 


\begin{tabular}{|c|c|r|r|}
\hline \hline superfield & gauge content & $Z_{2}$ & $Z_{2}^{\prime}$ \\
\hline$\left(N_{1}^{0}, E^{-}\right)$ & $(1,2,-1 / 2)$ & - & - \\
\hline$N_{2}^{0}$ & $(1,1,0)$ & - & - \\
$E^{+}$ & $(1,1,1)$ & - & - \\
\hline$\left(h_{3}^{0}, h_{3}^{-}\right)$ & $(1,2,-1 / 2)$ & + & - \\
$\left(h_{4}^{+}, h_{4}^{0}\right)$ & $(1,2,1 / 2)$ & + & - \\
\hline$\chi_{1}^{+}$ & $(1,1,1)$ & + & - \\
$\chi_{2}^{-}$ & $(1,1,-1)$ & + & - \\
\hline \hline
\end{tabular}

TABLE I. New superfields added to the MSSM to obtain radiative neutrino masses.

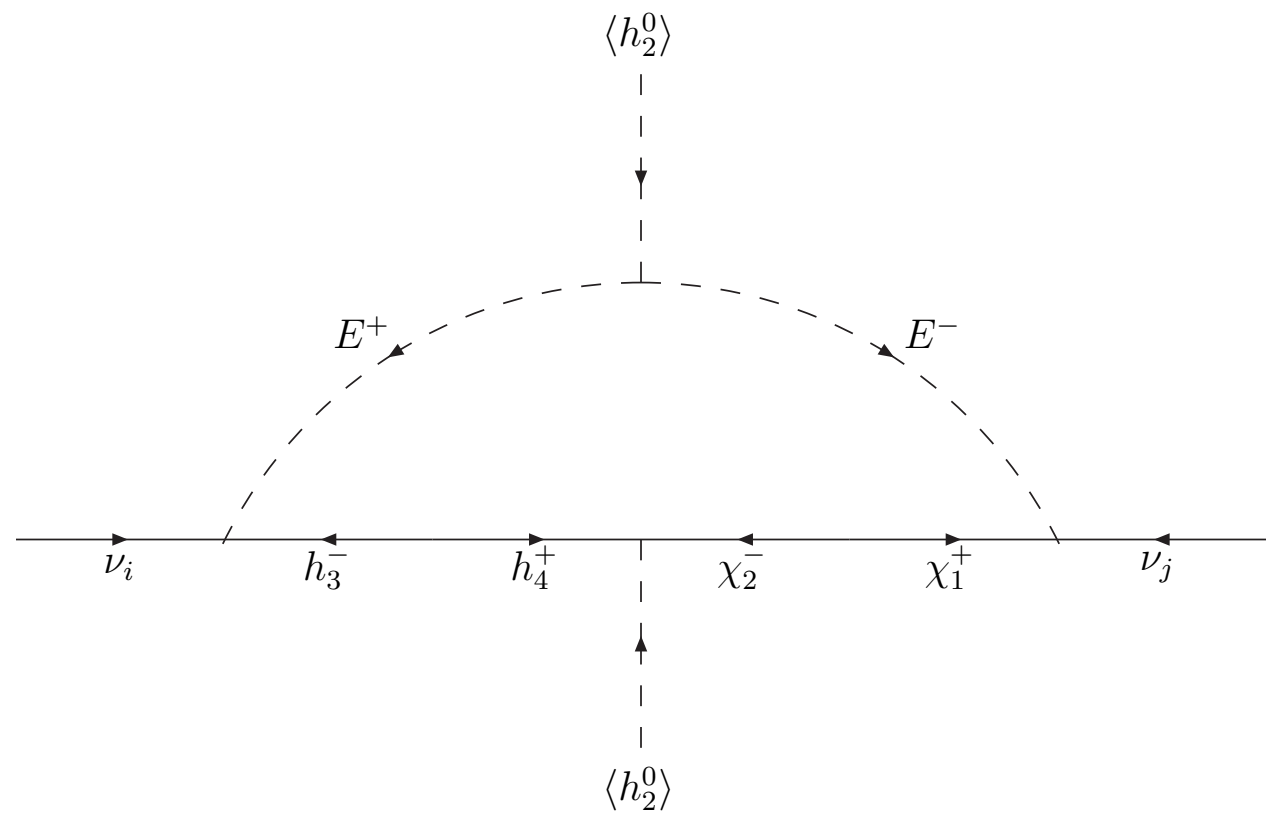

Fig. 1. One-loop radiative generation of neutrino masses. 